\title{
An Optimization System Applied in Optical Design of LED Light Guide Plate
}

\author{
Wen-Chin Chen ${ }^{1}$, Pen-Hsi Liou ${ }^{*}, 2$ and Bo-Jui Chen ${ }^{1}$
}

\author{
${ }^{I}$ Department of Industrial Management, Chung-Hua University, No. 707, Sec. 2, WuFu Rd., Hsinchu, Chinese Taipei, \\ Taiwan \\ ${ }^{2}$ Ph.D. Program of Technology Management, Chung-Hua University, No. 707, Sec. 2, WuFu Rd., Hsinchu, Chinese \\ Taipei, Taiwan
}

\begin{abstract}
Most of the small and medium-sized backlight modules are applied to a small amount of various products. Since the time to design a product is rigid and the light guide plate (LGP) is the key component that affects the quality of backlight modules, photovoltaic industries can enhance the core competence if they are able to develop high standard light guide plates with fast and efficient methods. The study proposes an optical optimal design system of light guide plate (LGP). The optimization design is conducted in the longitudinal structure of the LGP incidence plane with three LED light sources. Taguchi method is also used in carrying out the design of experiment through the TracePro, optical analysis software, and the experiment data which are employed as the back-propagation neural network (BPNN) training and testing samples and, then, created an optical quality predictor of the longitudinal structure. BPNN can predict the impact of incidence plane luminance versus the different constructed parameters. Finally, the optical quality predictor can effectively generate the optimal parameters settings combined with genetic algorithm (GA). The simulation results show that the proposed system can improve the non-uniformity problem of the incidence plane but also make it easier to design the longitudinal structure of the incidence plane.
\end{abstract}

Keywords: LGP, Taguchi method, BPNN, GA.

\section{INTRODUCTION}

In recent years, the internet and telecommunication have caused rapid development in many portable electronic products such as lap top computers, mobile phones, digital cameras, MPEG-4, tablet computers, and so on. Since liquid crystal display (LCD) has advantages of thin and lightweight, LCDs are strongly demanded to be widely applied to those equipments. LCD is the most popular flat panel display so far because of the most mature technology, the lowest cost, the most widespread application and the highest market share. An LCD is consisted of backlight modules, polarizing plates, a color filter, a glass substrate, a polyimide, liquid crystal materials, and a thin film transistor. LCD has to use a backlight source of either a cold cathode fluorescent lamp (CCFL) or a light-emitting diode (LED) to emit illuminants first. Those light sources pass through a polarizing plate and then cross liquid crystal materials in the way that the liquid crystal cell array changes the light angle in which the liquid crystal passes through. Before the light spreads across the LCD, the light source needs to pass through a color filter ahead and another polarizing plate. Finally, varying the biasing voltage of liquid crystal can

*Address correspondence to this author at the Ph.D. Program of Technology Management, Chung-Hua University, No. 707, Sec. 2, WuFu Rd., Hsinchu, Chinese Taipei, Taiwan; Tel: +886-4-27036678; Fax: +886-4-27036687; E-mail:ph.liou@msa.hinet.net control the light intensity and color such that various color combinations can be displayed on LCD.

In today's world, since the operating capability of computers has been increasing, many optical simulating software packages provide assisting tools such as TracePro, ASAP, LightTools, and SPEOS. Unlike the past methods which were time/manpower-consuming, CAE softwares can be used for simulation and analysis to save a great deal of time and money while designing a backlight module and an optical lens. In previous years, a trial-and-error or Taguchi method was usually applied to optical design. But, it was difficult to make it perfect. Besides, object models were needed to be created before those optical programs were undertaken for simulation analyses. Additionally, during the follow-up adjusting process, a new image had to be drawn after the size was modified. Then, the optical simulation was required to investigate the light distribution. A specimen simulation took about ten minutes per time and it had to be corrected several times that always spent too much time and cost for the whole process. The backlight module for smallsized products demands a little amount, variety, and tailormade needs. Moreover, the time to produce is strictly limited, it is the key to design an LGP which can meet the customer needs in a short period of time. For this reason, the industry and academic circles have been devoting themselves to doing research. They hope to bring out an optimization method in LGP design that will not only reduce developing time and cost for LCD industries and related businesses, 
thereof, but also improve competing capability of LCD industries.

Practical applications of LED light source of backlight module have been widely employed until now, particularly for small-sized panels. Since LED is a point light source pointing light-emitting face in the direction of LGP and having a light emission distribution in which the point light sources are scattered, it makes the LGP dot pattern design difficult. Since there were no specific simulation data for any specific incident angle, many literatures used design of experiment with optical simulation softwares to solve the problem. As a commonly used method to design goodquality LGP within default parameters, the Taguchi method has been adopted by many scholars to contribute to the design and adjustment of parameters of optical systems, which were employed for finding the optimal level of parameters [1-3]. To resolve this problem, many scholars used artificial neural networks and relevant optimization theories (SA, PSO, or GA) to obtain continuous global optimal parameter combinations, in which the optical quality in a complicated or nonlinear problem was exactly predicted and an optimal parameter combination was obtained with an integrated optimization theory [4-9]. Most of the previous studies focused on the dimension of the LGP dot pattern design or the LGP dot distribution. However, LED point light sources are scattered, which makes the LGP dot pattern design difficult. This problem can be solved by forming different incident planes of LGP and adjusting specific incident angle. Therefore, this study proposes an optical optimal design system of LGP. The optimization design is conducted in the longitudinal structure of the LGP incidence plane with three LED light sources. Two Taguchi experiments by using the TracePro software are carried out to obtain improved longitudinal structure. This study designs two longitudinal structures with V-cut and U-cut shapes. Two optical quality predictors are used to predict the luminance of different dimensions and shapes. Finally, each optical quality predictor is integrated with GA to find out the optical solution of design parameters for the different geometric shapes.

\section{DEVELOPMENT OF THE PROPOSED OPTICAL DESIGN SYSTEM}

Since there are no specific simulation data for any longitudinal structures of the light incidence plane in LGP, this study proposes an optical optimal design system of LGP. The optimization design is conducted in the longitudinal structure of the LGP incidence plane with three LED light sources. Two Taguchi experiments by using the TracePro software are conducted to improve the longitudinal structure. The quality characteristics are based on the uniformity of the light entering surface. This study designs two types of the longitudinal structures which are V-cut and U-cut. BPNN is used to train and test the experimental data to develop two optical quality predictors which are used to predict the luminance of different dimensions and shapes. Finally, each optical quality predictor is integrated with GA to find out the optical solution of design parameters for the different geometric shapes.

\subsection{Design and Implementation of the Experiment}

This study employs a commercial simulation software package called TracePro to design and analyze a 2.5-inch backlight module. The backlight module comprises an LGP, three light-emitting diodes, a light case, a reflector, and a frame. The LGP is a 54-mm x 42-mm x 1-mm-sized panel as shown in Fig. (1). The material of the LGP is made of PMMA (refractive index : 1.4935). The light sources are three 3-lumen SMD-type LEDs with sizes of 3-mm x 1-mm $x$ 1-mm placed at the short side of the LGP. The interval between the light source and the LGP is $0.6 \mathrm{~mm}$. They are even-distanced arranged and the half-intensity light angle is $120^{\circ}$ distributed by Lambertian light type. The refractive index of the light case and the reflector is 0.95 and the quantity of the simulation light beams is $6 \times 10^{5}$.

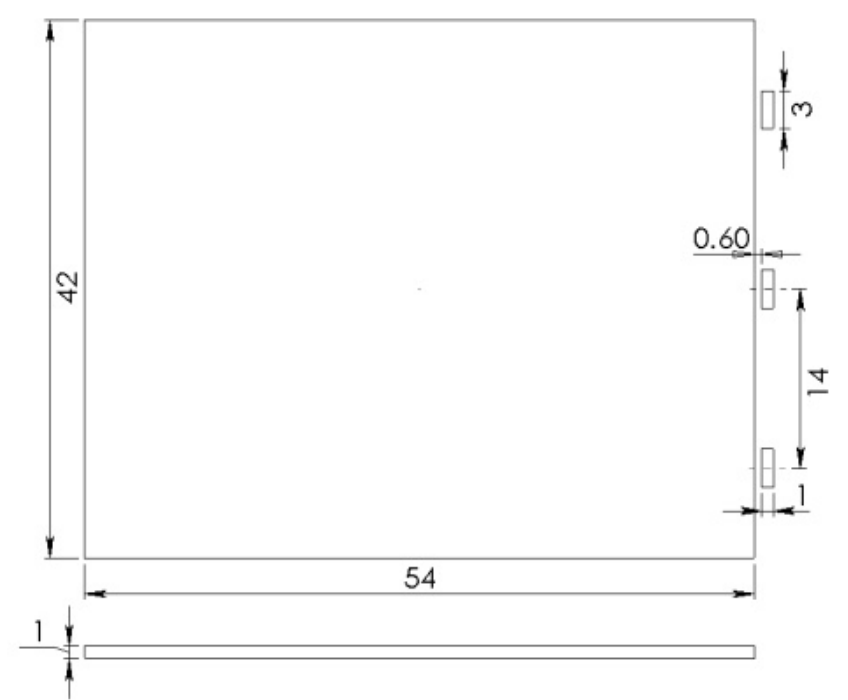

Fig. (1). Backlight module size.

\subsection{Parameter Formulation and Criteria Selection}

The initial parametric setting analysis is planned by using Taguchi method in which the impact of the luminance distribution on the light incident side of the LGP caused by the longitudinal structure on the light incident plane is explored. The quality characteristics are based on the uniformity of the light entering surface and are compared between both $\mathrm{V}$-cut and U-cut structures. For the V-cut structure, since different angles can make the light point to different directions and the depth and interval can affect the density distribution of the structure, these three factors (angle, depth, and interval) are chosen as the experimental criteria for the V-cut structure by using Taguchi method as shown in Fig. (2). As for the U-cut structure, the radius change can affect the curvature of its circular arc to vary the light point to different directions. The depth and interval can cause the density distribution of the structure. These three factors (radius, depth, and interval) are chosen as the experimental criteria for the U-cut structure by using Taguchi method as shown in Fig. (3). After the optical experiment has been analyzed and the engineers devoting themselves in optical design have been interviewed, the setting range for both $\mathrm{V}$-cut and U-cut structures is determined as listed in Tables $\mathbf{1}$ and $\mathbf{2}$. 


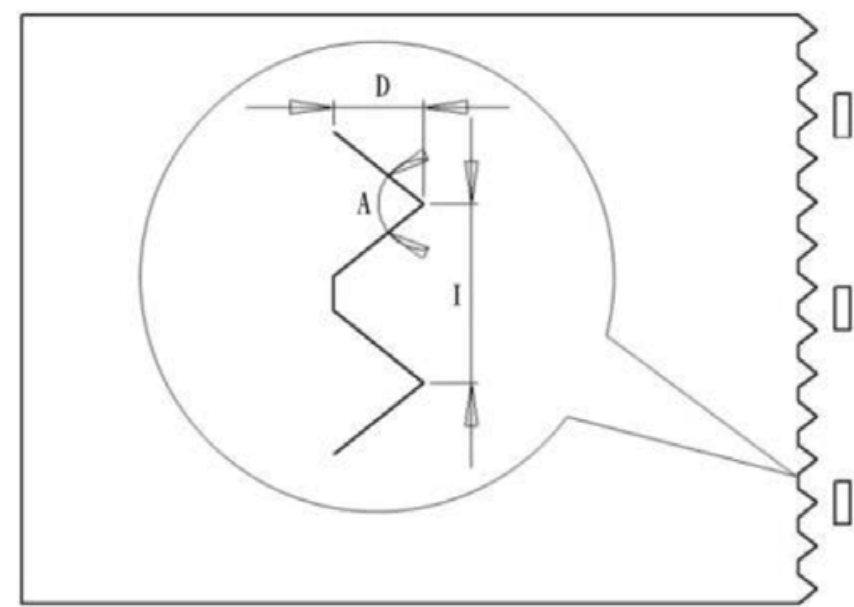

Fig. (2). Factors for V-cut structure.

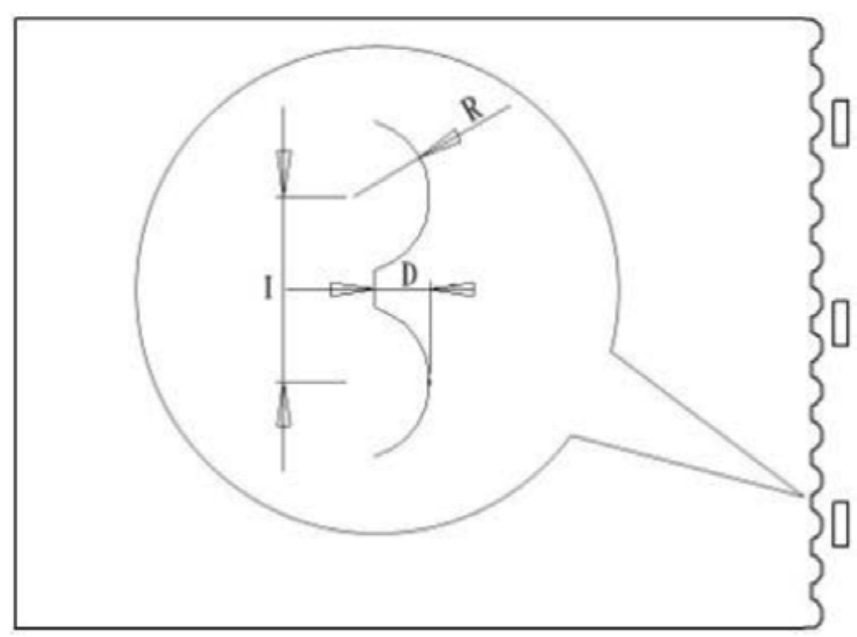

Fig. (3). Factors for U-cut structure.

Table 1. Range Setting for V-Cut

\begin{tabular}{|c|c|}
\hline Factors & Range Value \\
\hline \hline A: V-cut Angle & $75 \sim 135\left(^{\circ}\right)$ \\
\hline I : V-cut Interval & $0.09 \sim 0.13(\mathrm{~mm})$ \\
\hline D: V-cut Depth & $0.015 \sim 0.035(\mathrm{~mm})$ \\
\hline
\end{tabular}

Table 2. Range Setting for U-Cut

\begin{tabular}{|c|c|}
\hline Factors & Range Value \\
\hline \hline $\mathrm{R}:$ U-cut Radius & $0.03 \sim 0.05(\mathrm{~mm})$ \\
\hline I $:$ U-cut Interval & $0.09 \sim 0.13(\mathrm{~mm})$ \\
\hline D $:$ U-cut Depth & $0.015 \sim 0.035(\mathrm{~mm})$ \\
\hline
\end{tabular}

\subsection{Measurement of Quality Characteristics Experiment}

Experiment in this step applies the uniformity of the light incident surface to be one of the quality characteristics in the orthogonal array-based testing. The measurement methods are well defined to precisely show the quality characteristics. Before the decision of the measure methods, the luminance distribution after the refraction, reflection, and scattering of the three-LED light sources via the LGP is clearly explained. The experiment starts by the simulation of an LGP in which there are no microstructures on the light incident surface. It utilizes the uniformity of the dot pattern distribution in order to gain the luminance distribution on the light guide plate. For this reason, the radius of the dot pattern, $\mathrm{R}=0.1$, is set and the interval between $\mathrm{X}$ - and $\mathrm{Y}$-axes is 0.4 (hereinafter, also referred to this setting). After tracing the light rays, the luminance distribution of the light guide plate is shown in Fig. (4). The distributions of the brightness and the darkness are manifestly observed in the white outline shape. Therefore, this region is divided into 12 parts for measurement as indicated in Fig. (5). As to the uniformity, it is done by dividing the lowest and the highest luminances. By adopting this method, the uniformity on the light incident surface of the LGP and the data is listed in Table 3.

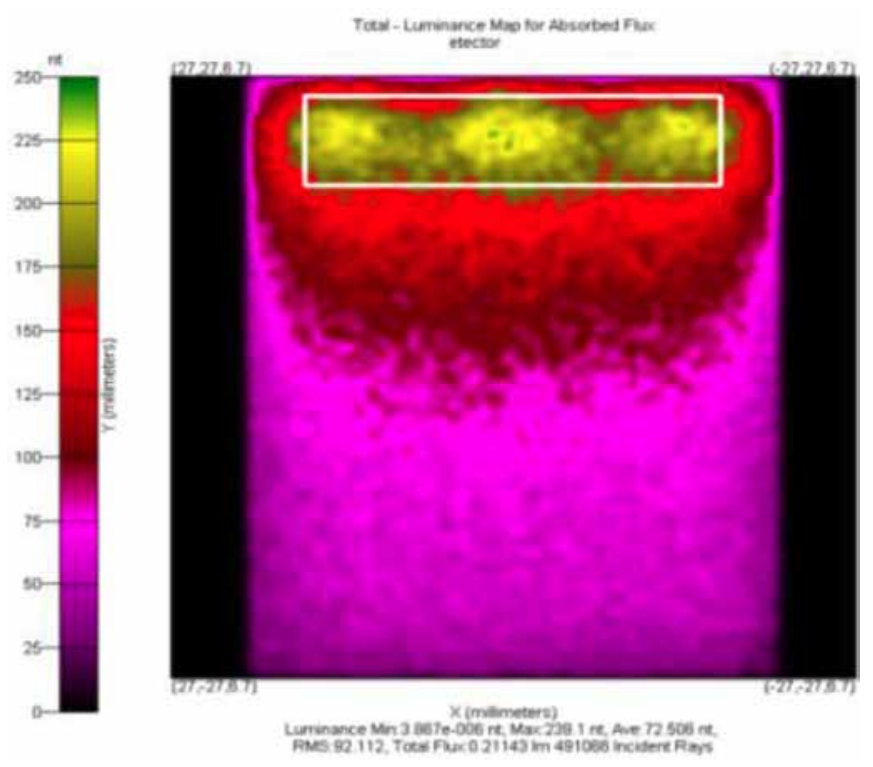

Fig. (4). Luminance distribution of the light guide plate without longitudinal structure on the light incident surface.

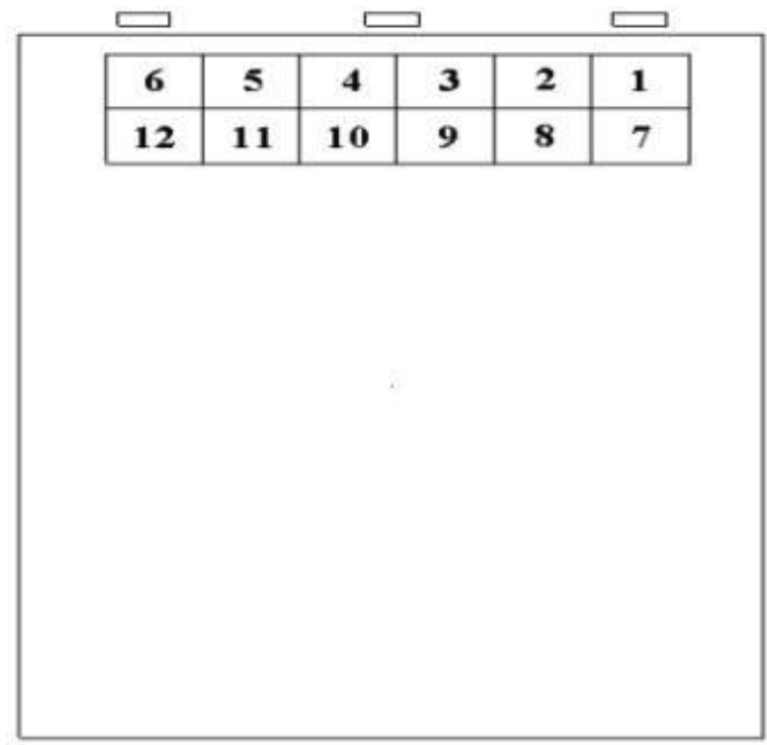

Fig. (5). The 12- measurement area on the light incident surface of the light guide plate. 
Table 3. The Luminance Value and the Uniform Value on the 12- Measurement Area

\begin{tabular}{|c|c|c|c|c|c|c|c|}
\hline $\mathbf{1}$ & $\mathbf{2}$ & $\mathbf{3}$ & $\mathbf{4}$ & $\mathbf{5}$ & $\mathbf{6}$ & Minimum & Uniform Value \\
\hline \hline 164.18 & 145.31 & 163.44 & 167.29 & 143.58 & 165.82 & $143.58\left(\mathrm{~cd} / \mathrm{m}^{2}\right)$ & Maximum \\
\hline $\mathbf{7}$ & $\mathbf{8}$ & $\mathbf{9}$ & $\mathbf{1 0}$ & $\mathbf{1 1}$ & $\mathbf{1 2}$ & $79.54 \%$ \\
\hline \hline 173.69 & 163.22 & 177.58 & 180.51 & 167.19 & 175.08 & $180.51\left(\mathrm{~cd} / \mathrm{m}^{2}\right)$ & \\
\hline
\end{tabular}

\subsection{Design of Experiment}

\subsubsection{First Taguchi Experiment}

Each range value of the V-cut and U-cut factors is evenly allocated into 5 levels as shown in Tables $\mathbf{4}$ and $\mathbf{5}$. Both the $\mathrm{V}$-cut and U-cut structures planned 3 factors and 5 levels and thereby a $L_{25}\left(5^{6}\right)$ orthogonal array testing is carried out. The parameters of the V-cut and U-cut are applied to the orthogonal array table, respectively. Two improved parameter settings of 25 experimental models are built for both the V-cut and U-cut structures. Thus, the luminance distribution of the light guide plate is obtained by simulating the TracePro software. Based on these two better parameter settings of 25 experimental results, the luminance distribution in the 12 area is measured and the uniformity of them is calculated. The results are indicated in Tables $\mathbf{6}$ and 7.

Table 4. Levels of V-Cut Factors in the First Taguchi Experiment

\begin{tabular}{|c|c|c|c|}
\hline Level & Angle $^{\circ}$ ) & Interval (mm) & Depth (mm) \\
\hline \hline 1 & 75 & 0.09 & 0.015 \\
\hline 2 & 90 & 0.1 & 0.02 \\
\hline 3 & 105 & 0.11 & 0.025 \\
\hline 4 & 120 & 0.12 & 0.03 \\
\hline 5 & 135 & 0.13 & 0.035 \\
\hline
\end{tabular}

Table 5. Levels of U-Cut Factors in the First Taguchi Experiment

\begin{tabular}{|c|c|c|c|}
\hline Level & Radius (mm) & Interval (mm) & Depth (mm) \\
\hline \hline 1 & 0.03 & 0.09 & 0.015 \\
\hline 2 & 0.035 & 0.1 & 0.02 \\
\hline 3 & 0.04 & 0.11 & 0.025 \\
\hline 4 & 0.045 & 0.12 & 0.03 \\
\hline 5 & 0.05 & 0.13 & 0.035 \\
\hline
\end{tabular}

\subsubsection{Second Taguchi Experiment}

According to the first Taguchi experiment, for the V-cut LGP, the $12^{\text {th }}$ set among the 25 experimental models represents the best uniformity of the light incident surface. As for the U-cut LGP, the $6^{\text {th }}$ set among the 25 experimental
Table 6. Uniformity of the Light Incident Surface Based on Levels of V-Cut Factors in the First Taguchi Experiment

\begin{tabular}{|c|c|c|c|c|}
\hline \multirow{2}{*}{$\begin{array}{l}\text { Experimental } \\
\text { Number }\end{array}$} & \multicolumn{3}{|c|}{ Factor } & \multirow{2}{*}{ Uniformity } \\
\hline & $\begin{array}{c}\text { V-Cut } \\
\left.\text { Angle ( }{ }^{\circ}\right)\end{array}$ & $\begin{array}{c}\text { V-Cut } \\
\text { Interval (mm) }\end{array}$ & $\begin{array}{c}\text { V-Cut } \\
\text { Depth (mm) }\end{array}$ & \\
\hline 1 & 75 & 0.09 & 0.015 & $82.73 \%$ \\
\hline 2 & 75 & 0.1 & 0.02 & $83.33 \%$ \\
\hline 3 & 75 & 0.11 & 0.025 & $85.29 \%$ \\
\hline 4 & 75 & 0.12 & 0.03 & $83.21 \%$ \\
\hline 5 & 75 & 0.13 & 0.035 & $84.90 \%$ \\
\hline 6 & 90 & 0.09 & 0.02 & $83.40 \%$ \\
\hline 7 & 90 & 0.1 & 0.025 & $85.47 \%$ \\
\hline 8 & 90 & 0.11 & 0.03 & $86.00 \%$ \\
\hline 9 & 90 & 0.12 & 0.035 & $84.34 \%$ \\
\hline 10 & 90 & 0.13 & 0.015 & $81.94 \%$ \\
\hline 11 & 105 & 0.09 & 0.025 & $85.12 \%$ \\
\hline$* 12$ & 105 & 0.1 & 0.03 & $86.99 \%$ \\
\hline 13 & 105 & 0.11 & 0.035 & $85.39 \%$ \\
\hline 14 & 105 & 0.12 & 0.015 & $83.32 \%$ \\
\hline 15 & 105 & 0.13 & 0.02 & $83.42 \%$ \\
\hline 16 & 120 & 0.09 & 0.03 & $84.61 \%$ \\
\hline 17 & 120 & 0.1 & 0.035 & $85.29 \%$ \\
\hline 18 & 120 & 0.11 & 0.015 & $83.32 \%$ \\
\hline 19 & 120 & 0.12 & 0.02 & $83.26 \%$ \\
\hline 20 & 120 & 0.13 & 0.025 & $82.13 \%$ \\
\hline 21 & 135 & 0.09 & 0.035 & $82.91 \%$ \\
\hline 22 & 135 & 0.1 & 0.015 & $82.10 \%$ \\
\hline 23 & 135 & 0.11 & 0.02 & $82.22 \%$ \\
\hline 24 & 135 & 0.12 & 0.025 & $83.69 \%$ \\
\hline 25 & 135 & 0.13 & 0.03 & $83.10 \%$ \\
\hline
\end{tabular}

models has shown the best uniformity of the light incident surface. Based on the parameter settings of these two experimental models, a half of the previous range setting is selected as a new level range setting for the second Taguchi experiment as shown in Tables $\mathbf{8}$ and $\mathbf{9}$. The experiments finding out the parameter for the longitudinal structure in size on the light incident surface are shown in Tables $\mathbf{1 0}$ and $\mathbf{1 1}$. 
Table 7. Uniformity of the Light Incident Surface Based on Levels of U-Cut Factors in the First Taguchi Experiment

\begin{tabular}{|c|c|c|c|c|}
\hline \multirow{2}{*}{$\begin{array}{c}\text { Experimental } \\
\text { Number }\end{array}$} & \multicolumn{3}{|c|}{ Factor } & \multirow{2}{*}{ Uniformity } \\
\hline & $\begin{array}{c}\text { U-Cut } \\
\text { Radius (mm) }\end{array}$ & $\begin{array}{c}\text { U-Cut } \\
\text { Interval (mm) }\end{array}$ & $\begin{array}{c}\text { U-Cut } \\
\text { Depth (mm) }\end{array}$ & \\
\hline 1 & 0.03 & 0.09 & 0.015 & $80.90 \%$ \\
\hline 2 & 0.03 & 0.1 & 0.02 & $81.83 \%$ \\
\hline 3 & 0.03 & 0.11 & 0.025 & $83.13 \%$ \\
\hline 4 & 0.03 & 0.12 & 0.03 & $82.94 \%$ \\
\hline 5 & 0.03 & 0.13 & 0.035 & $81.64 \%$ \\
\hline$* 6$ & 0.035 & 0.09 & 0.02 & $85.04 \%$ \\
\hline 7 & 0.035 & 0.1 & 0.025 & $82.84 \%$ \\
\hline 8 & 0.035 & 0.11 & 0.03 & $81.59 \%$ \\
\hline 9 & 0.035 & 0.12 & 0.035 & $83.03 \%$ \\
\hline 10 & 0.035 & 0.13 & 0.015 & $82.05 \%$ \\
\hline 11 & 0.04 & 0.09 & 0.025 & $81.69 \%$ \\
\hline 12 & 0.04 & 0.1 & 0.03 & $82.69 \%$ \\
\hline 13 & 0.04 & 0.11 & 0.035 & $83.01 \%$ \\
\hline 14 & 0.04 & 0.12 & 0.015 & $83.28 \%$ \\
\hline 15 & 0.04 & 0.13 & 0.02 & $82.33 \%$ \\
\hline 16 & 0.045 & 0.09 & 0.03 & $82.63 \%$ \\
\hline 17 & 0.045 & 0.1 & 0.035 & $82.41 \%$ \\
\hline 18 & 0.045 & 0.11 & 0.015 & $82.23 \%$ \\
\hline 19 & 0.045 & 0.12 & 0.02 & $82.37 \%$ \\
\hline 20 & 0.045 & 0.13 & 0.025 & $83.34 \%$ \\
\hline 21 & 0.05 & 0.09 & 0.035 & $83.24 \%$ \\
\hline 22 & 0.05 & 0.1 & 0.015 & $79.97 \%$ \\
\hline 23 & 0.05 & 0.11 & 0.02 & $82.49 \%$ \\
\hline 24 & 0.05 & 0.12 & 0.025 & $82.97 \%$ \\
\hline 25 & 0.05 & 0.13 & 0.03 & $84.07 \%$ \\
\hline
\end{tabular}

Table 8. Levels of V-Cut Factors in the Second Taguchi Experiment

\begin{tabular}{|c|c|c|c|}
\hline Level & Angle $\left(^{\circ}\right)$ & Interval (mm) & Depth (mm) \\
\hline \hline 1 & 90 & 0.09 & 0.025 \\
\hline 2 & 97.5 & 0.095 & 0.0275 \\
\hline 3 & 105 & 0.1 & 0.03 \\
\hline 4 & 112.5 & 0.105 & 0.0325 \\
\hline 5 & 120 & 0.11 & 0.035 \\
\hline
\end{tabular}

In view of the first Taguchi experiment, for the V-cut LGP, the $4^{\text {th }}$ model among the 25 experimental sets
Table 9. Levels of U-Cut Factors in the Second Taguchi Experiment

\begin{tabular}{|c|c|c|c|}
\hline Level & Radius (mm) & Interval (mm) & Depth (mm) \\
\hline \hline 1 & 0.03 & 0.08 & 0.015 \\
\hline 2 & 0.0325 & 0.085 & 0.0175 \\
\hline 3 & 0.035 & 0.09 & 0.02 \\
\hline 4 & 0.0375 & 0.095 & 0.0225 \\
\hline 5 & 0.04 & 0.1 & 0.025 \\
\hline
\end{tabular}

Table 10. Uniformity of the Light Incident Surface Based on Levels of V-Cut Factors in the Second Taguchi Experiment

\begin{tabular}{|c|c|c|c|c|}
\hline \multirow{2}{*}{$\begin{array}{l}\text { Experimental } \\
\text { Number }\end{array}$} & \multicolumn{3}{|c|}{ Factor } & \multirow{2}{*}{ Uniformity } \\
\hline & $\begin{array}{c}\text { V-Cut } \\
\text { Angle }\left({ }^{\circ}\right)\end{array}$ & $\begin{array}{c}\text { V-Cut } \\
\text { Interval (mm) }\end{array}$ & $\begin{array}{c}\text { V-Cut } \\
\text { Depth (mm) }\end{array}$ & \\
\hline 1 & 90 & 0.09 & 0.025 & $84.13 \%$ \\
\hline 2 & 90 & 0.095 & 0.0275 & $85.23 \%$ \\
\hline 3 & 90 & 0.1 & 0.03 & $86.75 \%$ \\
\hline$* 4$ & 90 & 0.105 & 0.0325 & $87.74 \%$ \\
\hline 5 & 90 & 0.11 & 0.035 & $87.53 \%$ \\
\hline 6 & 97.5 & 0.09 & 0.0275 & $85.73 \%$ \\
\hline 7 & 97.5 & 0.095 & 0.03 & $86.41 \%$ \\
\hline 8 & 97.5 & 0.1 & 0.0325 & $86.63 \%$ \\
\hline 9 & 97.5 & 0.105 & 0.035 & $86.24 \%$ \\
\hline 10 & 97.5 & 0.11 & 0.025 & $84.08 \%$ \\
\hline 11 & 105 & 0.09 & 0.03 & $82.87 \%$ \\
\hline 12 & 105 & 0.095 & 0.0325 & $87.05 \%$ \\
\hline 13 & 105 & 0.1 & 0.035 & $85.54 \%$ \\
\hline 14 & 105 & 0.105 & 0.025 & $83.62 \%$ \\
\hline 15 & 105 & 0.11 & 0.0275 & $84.19 \%$ \\
\hline 16 & 112.5 & 0.09 & 0.0325 & $84.12 \%$ \\
\hline 17 & 112.5 & 0.095 & 0.035 & $85.70 \%$ \\
\hline 18 & 112.5 & 0.1 & 0.025 & $83.23 \%$ \\
\hline 19 & 112.5 & 0.105 & 0.0275 & $84.65 \%$ \\
\hline 20 & 112.5 & 0.11 & 0.03 & $84.42 \%$ \\
\hline 21 & 120 & 0.09 & 0.035 & $85.07 \%$ \\
\hline 22 & 120 & 0.095 & 0.025 & $82.84 \%$ \\
\hline 23 & 120 & 0.1 & 0.0275 & $83.84 \%$ \\
\hline 24 & 120 & 0.105 & 0.03 & $85.53 \%$ \\
\hline 25 & 120 & 0.11 & 0.0325 & $85.01 \%$ \\
\hline
\end{tabular}

represents the best uniformity of the light incident surface. As compared with the best uniformity of the light incident 
Table 11. Uniformity of the Light Incident Surface Based on Levels of U-Cut Factors in the Second Taguchi Experiment

\begin{tabular}{|c|c|c|c|c|}
\hline \multirow{2}{*}{$\begin{array}{l}\text { Experimental } \\
\text { Number }\end{array}$} & \multicolumn{3}{|c|}{ Factor } & \multirow[b]{2}{*}{ Uniformity } \\
\hline & $\begin{array}{c}\text { U-Cut } \\
\text { Radius (mm) }\end{array}$ & $\begin{array}{c}\text { U-Cut } \\
\text { Interval (mm) }\end{array}$ & $\begin{array}{c}\text { U-Cut } \\
\text { Depth (mm) }\end{array}$ & \\
\hline 1 & 0.03 & 0.08 & 0.015 & $82.72 \%$ \\
\hline 2 & 0.03 & 0.085 & 0.0175 & $81.56 \%$ \\
\hline 3 & 0.03 & 0.09 & 0.02 & $82.57 \%$ \\
\hline 4 & 0.03 & 0.095 & 0.0225 & $82.04 \%$ \\
\hline 5 & 0.03 & 0.1 & 0.025 & $82.27 \%$ \\
\hline 6 & 0.0325 & 0.08 & 0.0175 & $81.72 \%$ \\
\hline$* 7$ & 0.0325 & 0.085 & 0.02 & $84.15 \%$ \\
\hline 8 & 0.0325 & 0.09 & 0.0225 & $82.72 \%$ \\
\hline 9 & 0.0325 & 0.095 & 0.025 & $80.89 \%$ \\
\hline 10 & 0.0325 & 0.1 & 0.015 & $81.58 \%$ \\
\hline 11 & 0.035 & 0.08 & 0.02 & $83.78 \%$ \\
\hline 12 & 0.035 & 0.085 & 0.0225 & $83.57 \%$ \\
\hline 13 & 0.035 & 0.09 & 0.025 & $83.17 \%$ \\
\hline 14 & 0.035 & 0.095 & 0.015 & $80.93 \%$ \\
\hline 15 & 0.035 & 0.1 & 0.0175 & $81.31 \%$ \\
\hline 16 & 0.0375 & 0.08 & 0.0225 & $82.80 \%$ \\
\hline 17 & 0.0375 & 0.085 & 0.025 & $83.08 \%$ \\
\hline 18 & 0.0375 & 0.09 & 0.015 & $80.80 \%$ \\
\hline 19 & 0.0375 & 0.095 & 0.0175 & $80.76 \%$ \\
\hline 20 & 0.0375 & 0.1 & 0.02 & $82.41 \%$ \\
\hline 21 & 0.04 & 0.08 & 0.025 & $83.49 \%$ \\
\hline 22 & 0.04 & 0.085 & 0.015 & $81.02 \%$ \\
\hline 23 & 0.04 & 0.09 & 0.0175 & $83.41 \%$ \\
\hline 24 & 0.04 & 0.095 & 0.02 & $83.06 \%$ \\
\hline 25 & 0.04 & 0.1 & 0.0225 & $82.00 \%$ \\
\hline
\end{tabular}

surface in the first Taguchi experiment, the result (uniformity: $87.74 \%$ ) has carried out $0.75 \%$ increase in the second time. As for the U-cut LGP, the $7^{\text {th }}$ model among the 25 experimental sets represents the best uniformity of the light incident surface. As compared with the best uniformity of the light incident surface in the previous investigation, the best uniformity is $84.15 \%$ which is decreased in the second Taguchi experiment. That is probably because the first best uniformity has been very close to the first best uniformity that causes the experimental set diverged from the best result while selecting the setting range from the lower half levels.

\subsection{Parameter Optimization}

\subsubsection{Development of the Optical Quality Predictor}

This research study utilizes the MATLAB software for numerical analysis and applies BPNN to V-cut LGP and U- cut LGP, respectively, to create optical quality predictors and further forecast the uniformity of the light incident surface. The results of the two orthogonal array tests can be employed as the training data of BPNN, where the three factors are for input data and the uniformity of the light incident surface is for output data. The experiment randomly adds five more test data to demonstrate the validity of BPNN in order to avoid the BPNN over training expresses in Tables 12 and 13.

Table 12. Test Data via Back-Propagation Neural Network (VCut)

\begin{tabular}{|c|c|c|c|c|}
\hline No. & Angle $\left(^{\circ}\right)$ & Interval (mm) & Depth (mm) & Uniformity \\
\hline \hline 1 & 130 & 0.12 & 0.02 & $83.76 \%$ \\
\hline 2 & 120 & 0.09 & 0.01 & $82.01 \%$ \\
\hline 3 & 80 & 0.14 & 0.03 & $82.68 \%$ \\
\hline 4 & 110 & 0.12 & 0.02 & $83.75 \%$ \\
\hline 5 & 70 & 0.1 & 0.03 & $83.04 \%$ \\
\hline
\end{tabular}

Table 13. Test Data via Back-Propagation Neural Network (UCut)

\begin{tabular}{|c|c|c|c|c|}
\hline No. & Radius (mm) & Interval (mm) & Depth (mm) & Uniformity \\
\hline \hline 1 & 0.03 & 0.1 & 0.03 & $81.23 \%$ \\
\hline 2 & 0.03 & 0.12 & 0.02 & $81.71 \%$ \\
\hline 3 & 0.035 & 0.085 & 0.0225 & $83.19 \%$ \\
\hline 4 & 0.04 & 0.1 & 0.02 & $82.12 \%$ \\
\hline 5 & 0.05 & 0.12 & 0.03 & $84.31 \%$ \\
\hline
\end{tabular}

After the V-cut data are given 13650 generations of training by using BPNN, the training and testing RMSE values are received of 0.0032 and 0.0594 , respectively. After 7851 generations of training given by U-cut data, the training and testing RMSE values are 0.0062 and 0.0509, respectively. The results have demonstrated that the RMSE value in the follow-up experiments needs to be in the acceptable range. The optical quality predictor trained by BPNN is expected to perform well for the uniformity of the light incident plane on LGP.

\subsubsection{GA Integrated with the Optical Quality Predictor}

Since Taguchi orthogonal array can merely optimize parameters for specific quality characteristics, this step associates genetic algorithm (GA) with the optical quality predictor created by BPNN to search for the optimal parameter of longitudinal structure for the uniformity of the light incident surface on LGP. While applying GA to gain the optimal parameter for both V-cut and U-cut LGPs, the best uniformity combinations of parameters in the two previous Taguchi methods plus and minus half of the initial setting range with levels is used to be the Upper Limit and the Lower Limit as shown in Tables 14 and 15. One-cutpoint method is used for the crossover procedure. It sets 100 to be the mating pool and 0.5 to be the mating rate. As for 
the mutation procedure, a single point mutation is adopted with the mutation rate of 0.06 . The convergence threshold was set to $10^{-6}$ or $10^{4}$ generations.

Table 14. GA Parameter Searching Range (V-Cut)

\begin{tabular}{|c|c|c|c|}
\hline \multirow{2}{*}{$\begin{array}{c}\text { Searching } \\
\text { Range }\end{array}$} & \multicolumn{3}{|c|}{ V-Cut Factor } \\
\cline { 2 - 4 } & Angle ( $\left.{ }^{\circ}\right)$ & Interval (mm) & Depth (mm) \\
\hline \hline Upper limit & 97.5 & 0.11 & 0.035 \\
\hline Lower limit & 82.5 & 0.1 & 0.03 \\
\hline
\end{tabular}

Table 15. GA Parameter Searching Range (U-Cut)

\begin{tabular}{|c|c|c|c|}
\hline \multirow{2}{*}{ Searching Range } & \multicolumn{3}{|c|}{ U-Cut Factor } \\
\cline { 2 - 4 } & Radius (mm) & Interval (mm) & Depth (mm) \\
\hline \hline Upper limit & 0.0375 & 0.095 & 0.0225 \\
\hline Lower limit & 0.0325 & 0.085 & 0.0175 \\
\hline
\end{tabular}

Since the crucial quality characteristic for both V-cut and U-cut LGPs is the uniformity of the light incident surface, the value is expected to be higher. The fitness function of $\mathrm{GA}$ is defined as the following:

$$
\operatorname{Min} F(X)=\left(1-U_{N}\right)^{2}
$$

s.t.

$$
L L_{i} \leq x_{i} \leq U L_{i} \quad i=1,2,3
$$

where $U_{N}$ is the quality predictor for the uniformity generated by BPNN and $\mathrm{X}=\left[\mathrm{x}_{1}, \mathrm{x}_{2}, \mathrm{x}_{3}\right]$ is the control parameter (factors). After combining GA with the optical quality predictor achieved by BPNN, the optimal parameters settings for V-cut and U-cut structured LGP are obtained illustrated in Table 16. After simulating by TracePro, the luminance distribution was shown in Figs. $(6,7)$, the result of the uniformity for V-cut is $88.19 \%$ as shown in Table $\mathbf{1 7}$. The result of the uniformity for U-cut is $85.22 \%$ as shown in Table 18.

Table 16. Optimal Parameter Setting for V-Cut and U-Cut

\begin{tabular}{|c|c|c|c|}
\hline Type & Angle $\left({ }^{\circ}\right)$ & Interval (mm) & Depth (mm) \\
\hline \hline V-cut & 91.2698 & 0.1065 & 0.0313 \\
\hline Type & Radius (mm) & Interval (mm) & Depth (mm) \\
\hline \hline U-cut & 0.0361 & 0.0889 & 0.0199 \\
\hline
\end{tabular}

Table 17. The Simulation Result of the Uniformity of V-Cut

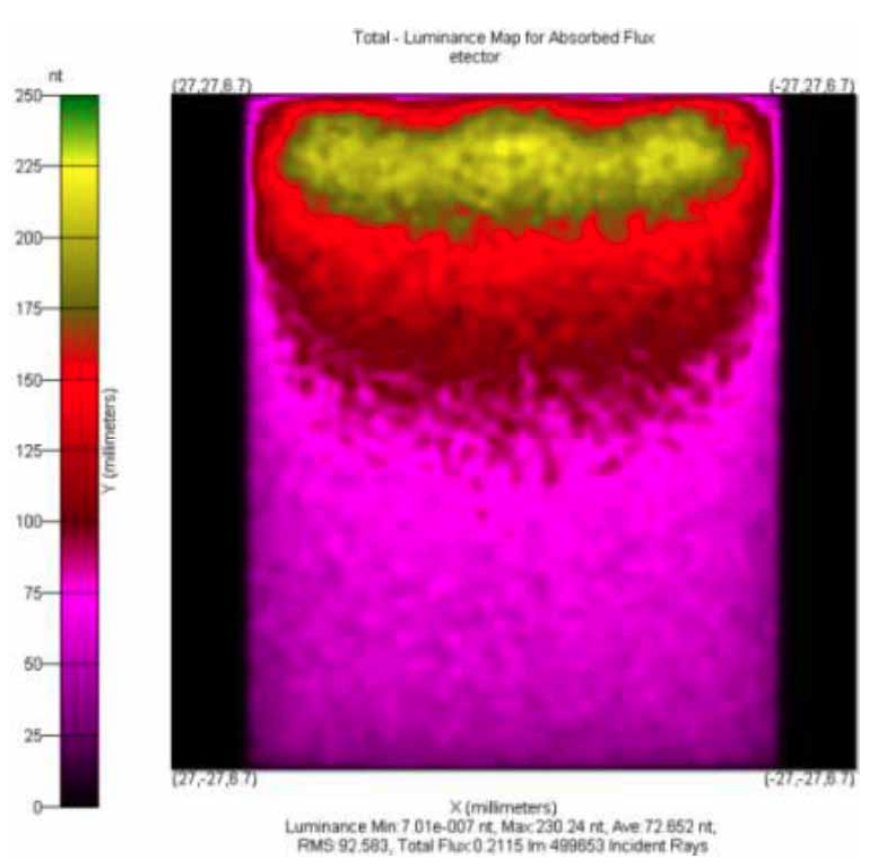

Fig. (6). The optimal parameters for the luminance of V-cut.

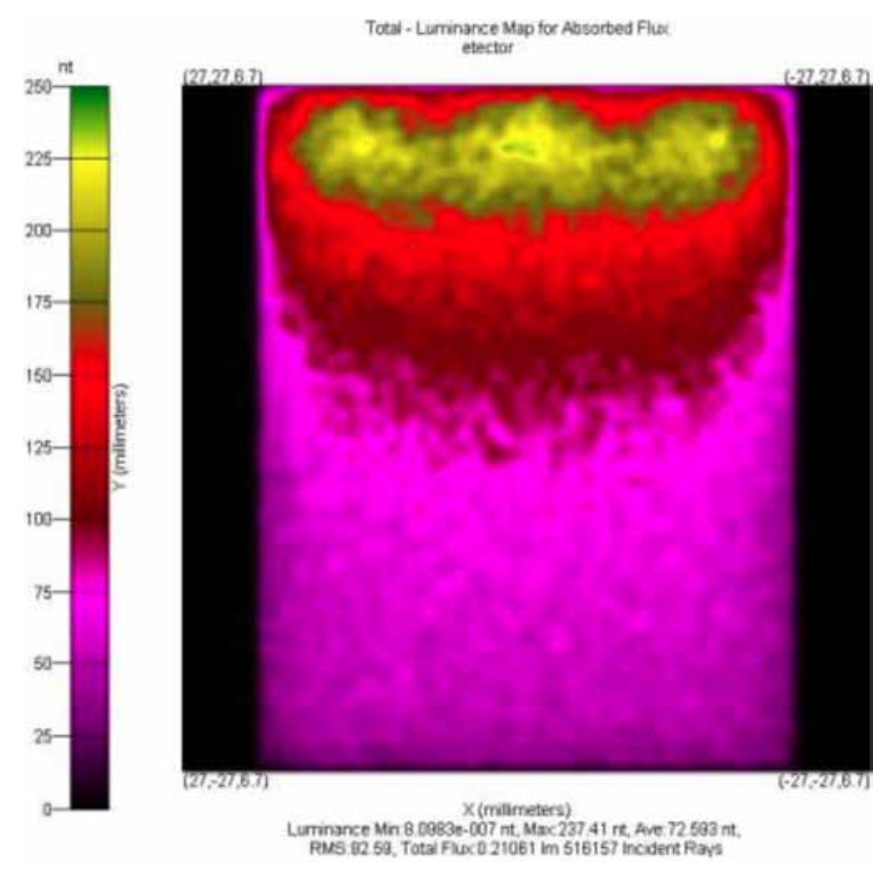

Fig. (7). The optimal parameters for the luminance of U-cut.

\section{RESULTS AND DISCUSSION}

This study proposes an optimization system that generates the optimal parameter settings for both V-cut and U-cut longitudinal structures. The results of V-cut are

\begin{tabular}{|c|c|c|c|c|c|c|c|}
\hline $\mathbf{1}$ & $\mathbf{2}$ & $\mathbf{3}$ & $\mathbf{4}$ & $\mathbf{5}$ & $\mathbf{6}$ & Minimum & Uniformity \\
\hline \hline 167.14 & 155.14 & 170.07 & 169.56 & 157.27 & 170.11 & $155.14\left(\mathrm{~cd} / \mathrm{m}^{2}\right)$ & \\
\hline $\mathbf{7}$ & $\mathbf{8}$ & $\mathbf{9}$ & $\mathbf{1 0}$ & $\mathbf{1 1}$ & $\mathbf{1 2}$ & Maximum \\
\hline \hline 169.93 & 174.56 & 175.81 & 175.91 & 172.83 & 166.38 & $175.91\left(\mathrm{~cd} / \mathrm{m}^{2}\right)$ & $88.19 \%$ \\
\hline
\end{tabular}


Table 18. The Simulation Result of the Uniformity of U-Cut

\begin{tabular}{|c|c|c|c|c|c|c|c|}
\hline $\mathbf{1}$ & $\mathbf{2}$ & $\mathbf{3}$ & $\mathbf{4}$ & $\mathbf{5}$ & $\mathbf{6}$ & Minimum & Uniformity \\
\hline \hline 171.98 & 151.69 & 172.69 & 174.84 & 151.33 & 171.43 & $151.33\left(\mathrm{~cd} / \mathrm{m}^{2}\right)$ & $\mathbf{1 2}$ \\
\hline $\mathbf{7}$ & $\mathbf{8}$ & $\mathbf{9}$ & $\mathbf{1 0}$ & $\mathbf{1 1}$ & $\mathbf{1 2}$ & Maximum & $85.22 \%$ \\
\hline \hline 169.25 & 170.98 & 176.95 & 177.58 & 169.85 & 170.07 & $177.58\left(\mathrm{~cd} / \mathrm{m}^{2}\right)$ & \\
\hline
\end{tabular}

analyzed as shown in Table $\mathbf{1 9}$ with the best uniformity processed by BPNN and GA of $88.19 \%$ which is $0.45 \%$ higher than that by the Taguchi method. The analysis of the U-cut structure is shown in Table 20. The optimal uniformity derived by BPNN and GA is $85.22 \%$ which is $0.18 \%$ higher than that by the Taguchi experiment. The finding shows the $\mathrm{V}$-cut structure is more efficient to solve the light uniformity problems than the U-cut structure used for the light incident surface of the LGP. It also lines the light exiting way for the LGP light incident surface and lowers the difficulty in the dot pattern design. Hence, the V-cut LGP is recommended for further research studies such as the dot pattern structure.

Table 19. The Optimal Parameter Settings (V-Cut)

\begin{tabular}{|c|c|c|c|c|}
\hline \multirow{2}{*}{$\begin{array}{c}\text { V-Cut } \\
\text { Structure }\end{array}$} & \multicolumn{3}{|c|}{ Factor } & \multirow{2}{*}{ Uniformity } \\
\cline { 2 - 5 } & $\begin{array}{c}\text { Angle } \\
\left({ }^{\circ}\right)\end{array}$ & $\begin{array}{c}\text { Interval } \\
(\mathbf{m m})\end{array}$ & $\begin{array}{c}\text { Depth } \\
(\mathbf{m m})\end{array}$ & \\
\hline \hline Taguchi method & 90 & 0.105 & 0.0325 & $87.74 \%$ \\
\hline BPNN+GA & 91.2698 & 0.1065 & 0.0313 & $88.19 \%$ \\
\hline
\end{tabular}

Table 20. The Optimal Parameter Settings (U-Cut)

\begin{tabular}{|c|c|c|c|c|}
\hline \multirow{2}{*}{$\begin{array}{c}\text { U-Cut } \\
\text { Structure }\end{array}$} & \multicolumn{3}{|c|}{ Factor } & \multirow{2}{*}{ Uniformity } \\
\cline { 2 - 5 } & $\begin{array}{c}\text { Radius } \\
(\mathbf{m m})\end{array}$ & $\begin{array}{c}\text { Interval } \\
(\mathbf{m m})\end{array}$ & $\begin{array}{c}\text { Depth } \\
(\mathbf{m m})\end{array}$ & \\
\hline \hline Taguchi method & 0.035 & 0.09 & 0.02 & $85.04 \%$ \\
\hline BPNN+GA & 0.0361 & 0.0889 & 0.0199 & $85.22 \%$ \\
\hline
\end{tabular}

\section{CONCLUSIONS}

This study proposes an optical design for the LED LGP. It carries out an optimal solution for the longitudinal structure on the light incident surface of the V-cut and U-cut LGPs. The experimental results are described below:

1. Both V-cut and U-cut structures on the light incident surface of the LGP can solve the problem about the uniformity of luminance distribution on the light incident surface. Based on the results of using Taguchi method, the V-cut structure has outperformed the U-cut structure.
2. The resulted data obtained by using Taguchi method are applied to BPNN for training and testing. A quality predictor for the light incident surface is then established. GA is finally integrated to find out the optimal parameter settings in which the uniform of luminance on the light incident surface can be improved to be $88.19 \%$ and $85.22 \%$ for V-cut and U-cut structures, respectively. These show better results than the those by using Taguchi method.

3. If the optimal $\mathrm{V}$-cut structure is used on the light incident surface, it makes a great change to the LED lighting method from point to line. It will simplify the further design on dot pattern structure.

\section{CONFLICT OF INTEREST}

The authors confirm that this article content has no conflict of interest.

\section{ACKNOWLEDGEMENTS}

Declared none.

\section{REFERENCES}

[1] W.C. Chen, M.Y. Tsai, and C.T. Chen, "Optimization design of LCD light guide plate", International Conference on Engineering and Business Management, Chengdu, China, Vol.7, pp. 4622-462h5, 2010.

[2] T.L. Su, H.W. Chen, C.F. Lu, "Systematic optimization for the evaluation of the microinjection molding parameters of light guide plate with TOPSIS-based Taguchi method", Adv. Polym. Technol., (Published online 19 May 2010), vol. 29, pp. 54-63, 2010

[3] Y.C. Fang, Y.F. Tzeng, and S.X. Li, "Multi-objective design and extended optimization for developing a miniature light emitting diode pocket-sized projection display", Opt. Rev., vol. 15, no. 5, 2010, pp. 241-250

[4] J. Smrelar, D. Pandit, M. Fast, M. Assadi, and S. De, "Prediction of power output of a coal-fired power plant by artificial neural network", Neural Comput. Appl., vol. 19, no. 5, pp.725-740, 2010.

[5] A. H. Gandomi and A. H. Alavi, "A new multi-gene genetic programming approach to nonlinear system modeling. Part I: materials and structural engineering problems", Neural Comput. Appl., vol. 21, no. 1, pp.171-187, 2012.

[6] C. J. Li, Y. C. Fang, and M. C. Cheng, "Study of optimization of an LCD light guide plate with neural network and genetic algorithm", Opt. Exp., vol. 17, no. 12, pp.10177-10188, 2009.

[7] W. C. Chen, T. T. Lai, M. W. Wang, and H. W. Hung, "An optimization system for LED lens design", Expert Syst. Appl., vol. 38, pp. 11976-11983, 2011.

[8] Y. C. Kim, T. S. OH, Y. M. Lee, "Optimized pattern design of lightguide plate (LGP) " Opt. Appl., XLI(4), pp. 863-872, 2011.

[9] C.J. Li, Y. C. Fang, W. T. Chu, and M. C. Cheng, "Design of a prism light-guide plate for an LCD backlight module", J. Soc. Inform. Display, published on line, 2012, DOI: 10.1889/1.2905041. 